\title{
Hybrid Tracers Based on Cyanine Backbones Targeting Prostate-Specific Membrane Antigen: Tuning Pharmacokinetic Properties and Exploring Dye-Protein Interaction
}

\author{
Albertus W. Hensbergen ${ }^{1}$, Tessa Buckle ${ }^{1}$, Danny M. van Willigen ${ }^{1}$, Margret Schottelius ${ }^{2}$, Mick M. Welling ${ }^{1}$, \\ Felicia A. van der Wijk ${ }^{1}$, Tobias Maurer ${ }^{3}$, Henk G. van der Poel ${ }^{4}$, Gabri van der Pluijm ${ }^{5}$, Wytske M. van Weerden ${ }^{6}$, \\ Hans-Jürgen Wester ${ }^{2}$, and Fijs W.B. van Leeuwen ${ }^{1}$ \\ ${ }^{I}$ Interventional Molecular Imaging Laboratory, Department of Radiology, Leiden University Medical Center, Leiden, The \\ Netherlands; ${ }^{2}$ Pharmazeutische Radiochemie, Technische Universität München, Garching, Germany; ${ }^{3}$ Martini-Klinik, \\ Universitätsklinikum Hamburg-Eppendorf, Hamburg, Germany; ${ }^{4}$ Department of Urology, Netherlands Cancer Institute-Antoni \\ van Leeuwenhoek Hospital, Amsterdam, The Netherlands; ${ }^{5}$ Department of Urology, Leiden University Medical Centre, Leiden, \\ The Netherlands; and ${ }^{6}$ Department of Urology, Erasmus MC Cancer Institute, Erasmus University Medical Center, Rotterdam, \\ The Netherlands
}

Prostate cancer surgery is currently being revolutionized by the use of prostate-specific membrane antigen (PSMA)-targeted radiotracers, for example, ${ }^{99 \mathrm{mT}}$ Tc-labeled PSMA tracer analogs for radioguided surgery. The purpose of this study was to develop a second-generation 99mTc-labeled PSMA-targeted tracer incorporating a fluorescent dye. Methods: Several PSMA-targeted hybrid tracers were synthesized: glutamic acid-urea-lysine (EuK)-Cy5- $\mathrm{mas}_{3}, \mathrm{EuK}\left(\mathrm{SO}_{3}\right) \mathrm{Cy} 5-\mathrm{mas}_{3}$, EuK-Cy5 $\left(\mathrm{SO}_{3}\right)-\mathrm{mas}_{3}$, EuK-(Ar)Cy5-mas ${ }_{3}$, and EuK-Cy5(Ar)-mas ${ }_{3}$; the Cy5 dye acts as a functional backbone between the EuK targeting vector and the 2-mercaptoacetyl-seryl-seryl-seryl $\left(\mathrm{mas}_{3}\right)$ chelate to study the dye's interaction with PSMA's amphipathic entrance funnel. The compounds were evaluated for their photophysical and chemical properties and PSMA affinity. After radiolabeling with 99mTc, we performed in vivo SPECT imaging, biodistribution, and fluorescence imaging on BALB/c nude mice with orthotopically transplanted PC346C tumors. Results: The dye composition influenced the photophysical properties (brightness range $0.3-1.5 \times 10^{4}$ $\mathrm{M}^{-1} \times \mathrm{cm}^{-1}$ ), plasma protein interactions (range $85.0 \% \pm 2.3 \%-$ $90.7 \% \pm 1.3 \%$ bound to serum, range $76 \% \pm 0 \%-89 \% \pm 6 \%$ stability in serum), PSMA affinity (half-maximal inhibitory concentration [ $\left[\mathrm{C}_{50}\right]$ range $19.2 \pm 5.8-175.3 \pm 61.1 \mathrm{nM}$ ) and in vivo characteristics (tumorto-prostate and tumor-to-muscle ratios range $0.02 \pm 0.00-154.73 \pm$ 28.48 and $0.46 \pm 0.28-5,157.50 \pm 949.17$, respectively; renal, splenic, and salivary retention). Even though all tracer analogs allowed tumor identification with SPECT and fluorescence imaging, ${ }^{99 m T c-E u K-~}$ $\left(\mathrm{SO}_{3}\right)$ Cy5-mas 3 had the most promising properties (e.g., half-maximal inhibitory concentration, $19.2 \pm 5.8$, tumor-to-muscle ratio, 5,157.50 \pm 949.17). Conclusion: Our findings demonstrate the intrinsic integration of a fluorophore in the pharmacophore in PSMA-targeted smallmolecule tracers. In this design, having 1 sulfonate on the indole moiety adjacent to EuK (99mTc-EuK-( $\left.\left.\mathrm{SO}_{3}\right) \mathrm{Cy} 5-\mathrm{mas}_{3}\right)$ yielded the most promising tracer candidate for imaging of PSMA.

Received Jul. 1, 2019; revision accepted Aug. 6, 2019.

For correspondence or reprints contact: Fijs W.B. van Leeuwen, Interventional Molecular Imaging Laboratory, Department of Radiology, Leiden University Medical Center, Albinusdreef 2, 2333 ZA Leiden, The Netherlands.

E-mail: f.w.b.van leeuwen@lumc.n

Published online Sep. 3, 2019.

COPYRIGHT (C 2020 by the Society of Nuclear Medicine and Molecular Imaging.
Key Words: prostate cancer; prostate-specific membrane antigen; hybrid tracers; image-guided surgery; cyanine

J Nucl Med 2020; 61:234-241

DOI: 10.2967/jnumed.119.233064

$\mathbf{P}$ rostate cancer, with an estimated 1.3 million new cases in 2018 ( $14 \%$ of the total number of new cases of cancer) and a 2018 estimate of over 350,000 deaths worldwide, is the second most frequent cancer and the fifth leading cause of cancer death in men (1). To eradicate the primary cancer, radical prostatectomyencompassing radical local resection, as well as dissection of lymphatic spread-is a commonly used surgical approach $(2,3)$. Although effective, this approach needs refinement and improvements, as recurrence rates after radical prostatectomy are still high (20\%-40\%) (4). In this scenario, prostate-tumor-specific tracers containing both a radioactive and an optical marker are highly warranted. The prostate-specific membrane antigen (PSMA), a transmembrane protein highly overexpressed on nearly all prostate cancer tumors (5), has received increased interest as a target for molecular imaging applications and, more recently, in image-guided surgical interventions $(6,7)$.

Clinical PSMA imaging reports generally describe the use of radiolabeled high-affinity targeting moieties based on glutamateurea, such as the inhibitor motif glutamic acid-urea-lysine (EuK) $(8,9)$, which are applied in a microdose regime $(\leq 100 \mu \mathrm{g} /$ patient $)$. The molecular binding interactions of glutamate-urea moieties to the PSMA protein have been studied extensively (10). The glutamateureido derivatives coordinate specifically with the binuclear zinc present deep within the PSMA protein. The amphipathic entrance funnel-that is, the funnel comprising the arene-binding site, arginine patch, and S1 accessory hydrophobic pocket-leads to this binding site (11). This S1 accessory hydrophobic pocket can be exploited as a secondary binding site (12). In line with this finding, it has been found that refinement of the backbone composition and length used to connect the imaging (or therapeutic) radiolabel to the 
glutamate-urea targeting vector impacts the PSMA binding in radiotracer development, exploiting charged, uncharged, or lipophilic spacers (13-15). Next to the development of PSMA-targeted agents for PET diagnostics (e.g., ${ }^{68} \mathrm{Ga}$-PSMA-HBED-CC (7)) or therapy (e.g., ${ }^{177} \mathrm{Lu}-\mathrm{PSMA}$ I\&T (16) or ${ }^{177} \mathrm{Lu}-\mathrm{PSMA}-617$ (17)), the SPECT agents ${ }^{111} \mathrm{In}$-Tc-PSMA I\&T and ${ }^{99 \mathrm{~m}}$ Tc-PSMA I\&S have successfully been used for the radioguided resection of metastatic lymph nodes in a salvage setting $(7,18,19) .{ }^{99 \mathrm{~m}} \mathrm{Tc}$ has proven to be the preferable radionuclide for the latter application because of its low costs, widespread availability, and short half-life (20).

The field of intraoperative fluorescence-guided surgery has been growing vigorously over the last 2 decades and is being used within the clinic more often because of its potential to realize radical resections while preserving healthy tissue $(2,21)$. With this reasoning, numerous fluorescence-based probes for the imaging of prostate cancer have been studied preclinically (22-24). Although the influence of various families of (commercially available) dyes on the PSMA-targeting ability has been studied (25), systematic refinement of the dye structure intending to tailor PSMA interaction has only been reported by Matsuoka et al., who reported that sulfonation of cyanine dyes had a positive influence on the PSMA affinity (26). In the development of fluorescent PSMA-targeted tracers, however, secondary binding to the amphipathic entrance funnel has also not received much interest.

Relying on fluorescence imaging alone during surgical guidance has its drawbacks: a limited penetration depth $(<10 \mathrm{~mm})$, the inability to roughly assess lesions or metastases preoperatively, tissue autofluorescence, and a high-dose-dependent detection accuracy (27). At the same time, the audible and numeric radioguidance provided by a $\gamma$-probe would benefit from being augmented with intraoperative optical identification of lesions. To overcome these shortcomings, a fluorescent and a radioactive label can be combined in a single molecule, creating so-called dual-labeled, bimodal, or rather hybrid tracers (28). This hybrid concept has also been adapted in preclinical PSMA imaging - for example, ${ }^{111}$ In-LICOR-800CW-LysDOTA-EuK (29); PSMA-11 derivatives (13); an ${ }^{18} \mathrm{~F}$-containing, Cy3-based probe (30); and PSMA imaging and fluorescence (PSMA I\&F) (31). Despite the ability of these tracer designs to offer a best-of-both-worlds scenario in image-guided surgery, in vivo analysis of the reported PSMA-targeted hybrid tracer analogs revealed that the synthetic designs are cleared and accumulated, at least fractionally, by the kidneys $(13,29,31)$. A drawback from the intraoperative use of these tracers is that their renal retention profile might lead to contamination of the surgical field with fluorescent urine and, as such, prohibit assessment of surgical margins (32).

To advance the concept of PSMA-targeted image-guided surgery, we aimed to design EuK-based, ${ }^{99 m}$ Tc-containing hybrid PSMA-targeted tracers with different substituents (sulfonate or benzene) that offer a highly selective tumor affinity, a high optical brightness and tissue penetration, favorable biodistribution, in vivo stability, and minimal (or fast) renal clearance. By adhering to the concept that the backbone can promote the pharmacophore interaction with the amphipathic entrance funnel of PSMA (13-15,33), we studied the influence of structural modifications on a cyanine dye (Fig. 1).

\section{MATERIALS AND METHODS}

The online supplemental materials (available at http://jnm.snmjournals.org) provide the complete, detailed synthetic procedures based on previously reported literature (34-38) and chemical analyses (matrix-assisted laser desorption/ionization time-of-flight and nuclear magnetic resonance [Supplemental Figs. 1-4] and high-performance liquid chromatography of hybrid tracers [Supplemental Figs. 5-9]).

\section{Photophysical Properties}

Photophysical properties (emissivity, quantum yield, and excitation and emission wavelengths $\left.\left[\lambda_{\mathrm{ex}} / \lambda_{\mathrm{em}}\right]\right)$ were assessed as based on earlier published procedures (39-41).

\section{Brightness}

The molar extinction coefficient of the hybrid tracers could not be determined because of their large molecular size. Therefore, the brightness of each hybrid tracer was calculated by multiplying its quantum yield by the emissivity of the corresponding free fluorophore (Supplemental Table 1).

\section{Radiochemistry}

The hybrid tracers were radiolabeled with ${ }^{99 \mathrm{~m}} \mathrm{Tc}$ for lipophilicity and in vivo experiments as follows: $3.90 \mu \mathrm{L}(6 \mathrm{nmol})$ of a $1.54 \mathrm{mM}$ hybrid tracer solution was mixed

with $8.13 \mu \mathrm{L}$ of $0.5 \mathrm{M}$ phosphate buffer, $\mathrm{pH} 8$;
FIGURE 1. (A) Schematic overview of working hypothesis, exemplified by hybrid tracer inside PSMA binding site with interactions between dye and amphipathic funnel. (B) Selected hybrid tracers with functionalization introduced on indole with different lipophilicities. (C) Hybrid tracer based on control Cy5 dye. (D) Chemical structures of EuK and 2-mercaptoacetyl-seryl-seryl-seryl ( mas $_{3}$ ) moieties. $\mathrm{S} 1$ and $\mathrm{S} 1$ ' are the primary binding sites of the protein. 
$12.03 \mu \mathrm{L}$ of $0.25 \mathrm{M}$ phosphate buffer, $\mathrm{pH} 8 ; 9.62 \mu \mathrm{L}$ of a $250 \mathrm{mg} / \mathrm{mL}$ solution of sodium L-tartrate in $0.5 \mathrm{M}$ phosphate buffer, $\mathrm{pH} 8$; and $2.41 \mu \mathrm{L}$ of a $4 \mathrm{mg} / \mathrm{mL}$ solution of stannous(II) chloride (freshly made and purged with helium) in a $3 \mathrm{mg} / \mathrm{mL}$ solution of L-ascorbic acid, which was prepared in a $10 \mathrm{mM} \mathrm{HCl}$ solution. After addition of $240 \mathrm{MBq}$ of ${ }^{99 \mathrm{~m}} \mathrm{Tc}$ in saline, the solution was heated to $90^{\circ} \mathrm{C}-95^{\circ} \mathrm{C}$ in capped vials for 20-30 min. The caps were then removed, and the solutions were cooled to room temperature for $10 \mathrm{~min}$. The degree of labeling (about 95\%) was determined by reversed-phase thin-layer chromatography using an eluent of $\mathrm{CH}_{3} \mathrm{CN}: \mathrm{H}_{2} \mathrm{O}$ (3:7), and the solutions were used without further purification.

\section{Lipophilicity}

The lipophilicity (the partition coefficient in octanol/water $[\log \mathrm{P}(\mathrm{o} / \mathrm{w})]$ ) was determined using previously published methods (9).

\section{Plasma Protein Binding (PPB)}

PPB was determined using previously published methods, with an $8 \mathrm{~K}$ Rapid Equilibrium Dialysis plate (product 90006; Sigma Aldrich) (40).

\section{Plasma Protein Stability}

The plasma protein stability was assessed using previously published methods (42).

\section{PSMA Affinity}

The affinity of the different tracers for PSMA (half-maximal inhibitory concentration $\left[\mathrm{IC}_{50}\right]$ ) was assessed using methods previously described by Weineisen et al. (9).

\section{Protein-Ligand Docking}

Protein-ligand docking interactions were carried out to understand the differences in the affinity findings and were calculated using AutoDock Vina, version 1.1.2 (43), and visualized using UCSF Chimera, version 1.31.1 (44), using the ligand-free human glutamate carboxypeptidase II (Protein Data Bank identification code 2OOT). After the protein had been prepared for docking in UCSF Chimera, the ligand molecule was selected and docking interactions were calculated. The most viable docking model was selected using the ViewDock plugin.

\section{Fluorescence Confocal Microscopy}

C4-2B $\mathrm{B}_{4}$ human prostate cells were cultured in RPMI-1640 medium enriched with $10 \%$ fetal bovine serum and penicillin/streptomycin (all Life Technologies Inc.) and $1 \mu \mathrm{M}$ enzalutamide (S1250; SelleckChem). Cells were kept under standard culturing conditions. For fluorescence confocal imaging, cells were seeded on glass-bottom culture dishes (MatTek Corp.) and placed in the incubator overnight. Confocal imaging was performed on viable cells, using an SP8 WLL fluorescence confocal microscope (Leica) (42). The cells were incubated with $1 \mu \mathrm{M}$ EuK-Cy5-mas 3 , EuK-( $\left.\mathrm{SO}_{3}\right) \mathrm{Cy} 5-\mathrm{mas}_{3}, \mathrm{EuK}-\mathrm{Cy} 5\left(\mathrm{SO}_{3}\right)-\mathrm{mas}_{3}$, EuK-(Ar) $\mathrm{Cy} 5-\mathrm{mas}_{3}$, or $\mathrm{EuK}-\mathrm{Cy} 5(\mathrm{Ar})-\mathrm{mas}_{3}$ for $1 \mathrm{~h}$ at $37^{\circ} \mathrm{C}$ and washed with phosphate-buffered saline 3 times. Prior to imaging, the nuclei of the cells were stained with Hoechst 33342 (ThermoFisher Scientific). Sequential scanning settings were applied to visualize all fluorescent features: Cy5 $\left(\lambda_{\text {ex }}, 633 \mathrm{~nm} ; \lambda_{\mathrm{em}}, 650-700 \mathrm{~nm}\right)$ and Hoechst $\left(\lambda_{\mathrm{ex}}, 405 \mathrm{~nm} ; \lambda_{\mathrm{em}}\right.$, $420-470 \mathrm{~nm}$ ). Fluorescence intensities were determined using previously used methods (45).

\section{In Vivo Tumor Model}

PC346C cells were washed with Hanks balanced salt solution (Invitrogen) and suspended in a 1:1 mixture of Hanks balanced salt solution and Matrigel (VWR) before injection. Prepared PC346C cells (46) were orthotopically transplanted into the prostate of male BALB/ c nude mice (Harlan; 6-8 wk old). Before transplantation, the mice were anesthetized using a 1:1:2 solution of fentanyl-fluanisone (Hypnorm; VetaPharma Ltd.), midazolam (Dormicum; Roche), and $\mathrm{H}_{2} \mathrm{O}$ ( $5 \mu \mathrm{L} / \mathrm{g}$, administered intraperitoneally). The prostate was located via a small incision in the abdominal wall, after which $2.0 \times 10^{6} \mathrm{PC} 346 \mathrm{C}$ cells (in $20 \mu \mathrm{L}$ of 1:1 Hanks balanced salt solution/Matrigel) were injected into the prostate. After repositioning of the prostate, the incision was closed. Pain relief was given via subcutaneous injection of $10 \mu \mathrm{L}$ of buprenorphine (Temgesic, $5 \mathrm{mg} / \mathrm{mL}$; Actavis) in $0.5 \mathrm{~mL}$ of $0.90 \%(\mathrm{w} / \mathrm{v}) \mathrm{NaCl}$ in $\mathrm{H}_{2} \mathrm{O}$, at $30 \mathrm{~min}$ before and $24 \mathrm{~h}$ after the procedure. Approximately $2 \mathrm{wk}$ after transplantation, palpable lesions were deemed suitable for further experiments when $2-5 \mathrm{~mm}$ in diameter. All animal experiments were approved by the local ethics committee of the Leiden University Medical Center before execution. The experiments were performed in accordance with the Experiments on Animals Act (Wet op de Dierproeven, 2014), which is the applicable legislation in The Netherlands in accordance with the European guidelines (EU directive 2010/63/EU) regarding the protection of animals used for scientific purposes. All experiments were executed in an establishment licensed for the use of experimental animals (Leiden University Medical Center).

\section{In Vivo SPECT Imaging and Quantitative Assessment of Biodistribution}

For biodistribution $(n=4)$ and for SPECT imaging $(n=2)$, approximately $40 \mathrm{MBq}$ were injected intravenously. At $2 \mathrm{~h}$ after injection, SPECT imaging was performed followed by ex vivo evaluation of the biodistribution (in total, 6 animals per tracer). Total-body imaging and data reconstruction for PC346C-tumor-bearing mice was performed on a U-SPECT scanner (MILabs) using previously described methods $(42,47)$.

After imaging, the animals were sacrificed by cervical dislocation and various tissues were resected, weighed, and counted for radioactivity. Quantitative biodistribution measurements (percentage injected dose per gram of tissue [\% ID/g] and tumor-to background ratios $[(\% \mathrm{ID} / \mathrm{g}$ tumor $) /(\% \mathrm{ID} / \mathrm{g}$ background] $)$ were calculated.

\section{Ex Vivo Fluorescence Imaging}

Directly after SPECT imaging, the mice were sacrificed and fluorescence imaging was performed using a clinical-grade laparoscope (Karl Storz Endoskope $\mathrm{GmbH}$ ) (48). Ex vivo images of excised tissues were acquired at $\mathrm{Cy} 5\left(\lambda_{\mathrm{ex}}, 640 \mathrm{~nm} ; \lambda_{\mathrm{em}}, 680 \mathrm{~nm}\right)$. For imaging with the fluorescence laparoscope, the tumor was placed on top of a heightadjustable lift and imaged with a setup identical to one described previously $(42,48)$. Images were acquired using a clinical-grade Image $1 \mathrm{~S}$ camera system (Karl Storz Endoskope $\mathrm{GmbH}$ ) equipped with a $0^{\circ}$ laparoscope. Excitation was achieved using a Cy5-modified D-Light C light source (Karl Storz Endoskope $\mathrm{GmbH}$ ). Cy5 was detected by placement of an additional standard eyepiece adaptor (catalog number 20100034; Karl Storz Endoskope GmbH) between the camera and the laparoscope $(42,48)$.

\section{Statistical Data Analysis}

Analytical data were expressed as mean $\pm \mathrm{SD}$ as calculated using GraphPad Prism, version 7. The significance of 2 mean values was calculated using a Student $t$ test or ANOVA. Outliers were detected and removed using the Grubbs method. The level of significance was set at a $P$ value of 0.05 or less.

\section{RESULTS}

\section{Chemistry and Photophysical Properties}

The supplemental materials show the results for the chemistry and photophysical properties of the hybrid tracers.

\section{Lipophilicity and Plasma Protein Interaction}

The partition coefficient in octanol/water indicated that the site of functionalization slightly affected the lipophilicity (Table 1). As a first indication for in vivo behavior, the degree of PPB was 
studied, yielding the following trend: EuK-Cy5-mas ${ }_{3}<$ EuK$\left(\mathrm{SO}_{3}\right) \mathrm{Cy} 5-\mathrm{mas}_{3} \approx \mathrm{EuK}-\mathrm{Cy} 5\left(\mathrm{SO}_{3}\right)-\mathrm{mas}_{3}<\mathrm{EuK}-\mathrm{Cy} 5(\mathrm{Ar})-\mathrm{mas}_{3}<$ EuK-(Ar)Cy5-mas 3 (Table 1). It is interesting that the site of the substitution with a benzene moiety seemed to influence PPB to a statistically significant degree $(81.7 \% \pm 1.4 \%$ bound for EuKCy5(Ar)- mas $_{3}$ vs. $88.6 \% \pm 2.5 \%$ bound for EuK-(Ar)Cy5-mas 3 ; $P=0.0031)$, a change that could translate to differences in an in vivo setting (49). A correlation $(R=0.9232, P=0.0252$; Supplemental Fig. 11) between lipophilicity and PPB was observed: an increase in lipophilicity led to a higher PPB, as is in line with the literature (31).

Besides PPB, the chemical stability in plasma proteins was a critical feature in tracer performance $(41,49)$. After incubation in serum at $37^{\circ} \mathrm{C}$ for $24 \mathrm{~h}$, the absorbance intensity decreased (range, $75 \% \pm 0 \%-97 \% \pm 2 \% ; P>0.05$; Table 1$)$. The decrease in fluorescence signal was in line with the decrease in absorbance (range, $76 \% \pm 0 \%-89 \% \pm 6 \%$; Table 1). The ${ }^{99 \mathrm{~m}} \mathrm{Tc}-\mathrm{mas}_{3}$ complex was stable in all tracers (Supplemental Table 2), as previously established (9). These findings thus indicated that the tracers are sufficiently stable.

\section{Fluorescence Confocal Microscopy and Receptor Affinity}

In vitro imaging revealed an influence of the dye composition on the cellular binding; EuK- $\left(\mathrm{SO}_{3}\right) \mathrm{Cy} 5-\mathrm{mas}_{3}$ portrayed the highest binding on PSMA-overexpressing LNCaP cells, whereas the symmetric EuK-Cy5-mas 3 showed the least binding (Figs. 2A and 2B). Blocking with $((S)$-1-carboxy-5-(4-(iodo-benzamido)pentyl) carbamoyl)-l-glutamic acid $(10 \mu \mathrm{M})$ resulted in a significant reduction in tracer uptake (Fig. 2F). Blocking was most efficient for EuK- $\left(\mathrm{SO}_{3}\right) \mathrm{Cy} 5-\mathrm{mas}_{3}$ and EuK-Cy5 $\left(\mathrm{SO}_{3}\right)-\mathrm{mas}_{3}(\leq 90 \%)$, indicating that these tracers had the lowest amount of nonspecific binding.

Assessment of the binding affinity (half-maximal inhibitory concentration; Figs. 2A-2E) revealed a unique influence of the cyanine backbone composition on the affinity for PSMA. EuK- $\left(\mathrm{SO}_{3}\right)$ Cy5- mas $_{3}$ had a high affinity for PSMA $(19.2 \pm 5.8 \mathrm{nM})$, an affinity that was in the same range as that of clinically used tracers

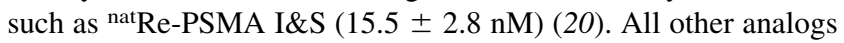
had a lower affinity (Figs. 2A-2E); it thus seems that not only the introduction of a sulfonate moiety but also its location had an effect on the affinity. This increase could be the result of favorable interactions of the ligand with the protein. Protein-ligand docking indeed suggested possible interactions between the cyanine backbone and the amphipathic funnel, indicating that the fluorophore can become an integral part of the pharmacophore (Supplemental Fig. 12). In correspondence with the affinity results, docking calculations for the other 4 hybrid tracers did not yield this favored configuration, underlining the importance of functional chemical moieties and their placement.

\section{In Vivo Imaging and Biodistribution}

In vivo SPECT imaging at $2 \mathrm{~h}$ after administration of $1 \mathrm{nmol}$ of tracer revealed clear differences in tracer uptake and distribution (Fig. 3). Tumors were visible for all tracers, and tracer retention in the kidneys was measured for all compounds. The more lipophilic tracers, ${ }^{99 \mathrm{~m}} \mathrm{Tc}-\mathrm{EuK}-(\mathrm{Ar}) \mathrm{Cy} 5-\mathrm{mas}_{3}$ and ${ }^{99 \mathrm{~m}} \mathrm{Tc}-\mathrm{EuK}-\mathrm{Cy} 5(\mathrm{Ar})-\mathrm{mas}_{3}$, showed higher retention in the liver and bowels than did the other tracers. Quantitative assessment of the biodistribution ex vivo after counting of radioactivity and weighing of the excised tissues revealed biodistribution patterns comparable to those of the SPECT images (Supplemental Table 3).

Uptake in the kidneys after $2 \mathrm{~h}$ was in the range of $8.98 \pm 5.78$ $18.35 \pm 8.51 \% \mathrm{ID} / \mathrm{g}$ (Supplemental Table 3). Other notorious locations for PSMA tracer accumulation, the salivary glands and the spleen (which are essentially PSMA-negative in mice) (31), portrayed low uptake as well (range $0.02 \pm 0.01-0.23 \pm 0.12$ $\% \mathrm{ID} / \mathrm{g}$ for salivary glands and range $0.18 \pm 0.19-1.03 \pm 0.72$ $\% \mathrm{ID} / \mathrm{g}$ for spleen; $P>0.05$ for all comparisons). Chemical alterations on the cyanine backbone did not induce statistically significant organ uptake changes $(P>0.05)$. As expected, hybrid tracer uptake in the brain was almost undetectable, indicating that the tracers were not able to cross the blood-brain barrier.

Quantitative assessment of the biodistribution further revealed the highest accumulation in tumorous tissue for ${ }^{99 \mathrm{~m}} \mathrm{Tc}-\mathrm{EuK}-\left(\mathrm{SO}_{3}\right)$ Cy5-mas 3 (15.27 $\pm 2.85 \%$ ID/g; Supplemental Table 3). The other tracers had lower tumor uptake and were in the same range $(P>$ 0.05). Blood uptake values seemed to be somewhat in line with lipophilicity; the more lipophilic a tracer was, the higher was its blood retention value (Table 1; Supplemental Table 3). ${ }^{99 \mathrm{~m}} \mathrm{Tc}-$ EuK- $\left(\mathrm{SO}_{3}\right) \mathrm{Cy} 5-\mathrm{mas}_{3}$ and ${ }^{99 \mathrm{~m}} \mathrm{Tc}-\mathrm{EuK}-\mathrm{Cy} 5\left(\mathrm{SO}_{3}\right)-\mathrm{mas}_{3}$, the most hydrophilic tracers, thus provided the lowest blood retention at $2 \mathrm{~h}$ (Supplemental Table 3). Another important quantification for

TABLE 1

Physical and Photophysical Characteristics of PSMA-Targeted Hybrid Tracers

\begin{tabular}{|c|c|c|c|c|c|c|}
\hline Compound & $\begin{array}{c}\lambda_{\text {ex,max }} / \lambda_{\text {em,max }} \\
\text { in } \mathrm{H}_{2} \mathrm{O} / \mathrm{PBS}(\mathrm{nm})\end{array}$ & $\begin{array}{c}\Phi_{\mathrm{F}} \\
(\% \text { in PBS) }\end{array}$ & $\begin{array}{l}\text { Brightness } \\
\left(\times 10^{4} \mathrm{M}^{-1} \times\right. \\
\left.\mathrm{cm}^{-1} \text { in PBS }\right)^{\dagger}\end{array}$ & $\begin{array}{c}\text { Lipophilicity } \\
\left(\log \mathrm{P}_{(\mathrm{o} / \mathrm{w})} ; n=6\right)^{\star}\end{array}$ & $\begin{array}{c}\text { PPB } \\
(n=3)\end{array}$ & $\begin{array}{l}\text { Serum stability after } 24 \mathrm{~h} \\
(\% \text { remaining; } n=2)\end{array}$ \\
\hline EuK-Cy5-mas 3 & $644 / 663(18)^{\ddagger}$ & 12 & 1.1 & $-2.13 \pm 0.10$ & $88.3 \pm 1.6$ & Abs: $80 \pm 6 /$ Flu: $86 \pm 5$ \\
\hline EuK-(SO 3$)$ Cy5-mas 3 & $648 / 664(16)^{\ddagger}$ & 15 & 1.4 & $-2.86 \pm 0.05$ & $85.0 \pm 2.3$ & Abs: $97 \pm 2 /$ Flu: $89 \pm 6$ \\
\hline EuK-Cy5( $\left(\mathrm{SO}_{3}\right)-\mathrm{mas}_{3}$ & $648 / 664(16)^{\ddagger}$ & 10 & 1.5 & $-2.70 \pm 0.01$ & $87.4 \pm 2.0$ & Abs: $82 \pm 7 /$ Flu: $85 \pm 1$ \\
\hline EuK-(Ar)Cy5-mas 3 & $664 / 682(18)^{\ddagger}$ & 9 & 0.3 & $-1.75 \pm 0.10$ & $90.7 \pm 1.3$ & Abs: $85 \pm 3 /$ Flu: $79 \pm 1$ \\
\hline EuK-Cy5(Ar)-mas 3 & $663 / 680(17)^{\ddagger}$ & 12 & 0.7 & $-1.84 \pm 0.06$ & $89.0 \pm 2.5$ & Abs: $75 \pm 0 /$ Flu: $76 \pm 0$ \\
\hline
\end{tabular}

\footnotetext{
${ }^{\dagger}$ Calculated using molecular extinction coefficient of free fluorophore.

*Measured using ${ }^{\text {}}$ M9mTc-labeled hybrid tracers.

${ }^{\ddagger}$ Parenthetic numbers are Stokes shift.

$\lambda_{\mathrm{ex}, \max } / \lambda_{\mathrm{em} \text { max }}=$ maximum $\lambda_{\mathrm{ex}} / \lambda_{\mathrm{em}} ; \Phi_{\mathrm{F}}=$ quantum yield; log $\mathrm{P}_{(\mathrm{o} / \mathrm{w})}=$ the partition coefficient in octanol/water; PBS $=$ phosphatebuffered saline; Abs = absorbance; Flu = fluorescence.
} 


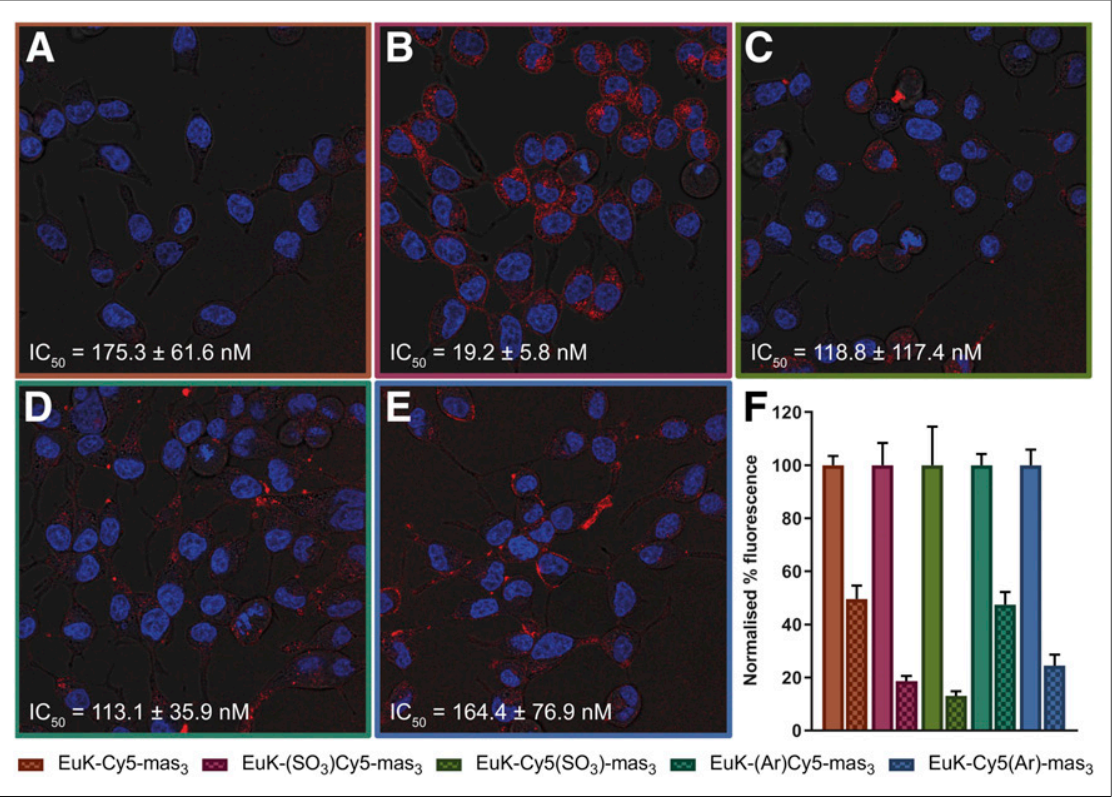

FIGURE 2. In vitro assessment of hybrid tracers. Shown is staining of extracellular expression of PSMA on $\mathrm{C}^{2}-2 \mathrm{~B}_{4}$ cells after incubation with nuclear stain (Hoechst; in blue) and hybrid tracer (Cy5; in red), with half-maximal inhibitory concentrations ( $\left(\mathrm{C}_{50}\right.$, measured with ${ }^{125}$-EuK-I-BA [((S)-1carboxy-5-(4-(iodo-benzamido)pentyl)carbamoyl)-L-glutamic acid] as competitive radioligand). (A)

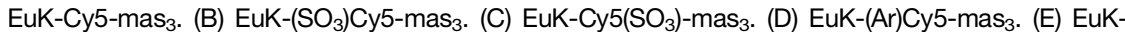
Cy5(Ar)-mas ${ }_{3}$. (F) Blocking (checkered) of PSMA with EuK-I-BA. Binding affinities are represented as mean $\pm \mathrm{SD}$.

assessing tracer performance was the tumor-to-organ ratio for different tissues, as the tumors were well vascularized and surrounded by muscle and prostate tissue. Therefore, the tumor uptake was related to the background uptake in blood, muscle, prostate, and fat (Supplemental Table 3). Overall, ${ }^{99 \mathrm{~m}} \mathrm{Tc}-\mathrm{EuK}-\left(\mathrm{SO}_{3}\right) \mathrm{Cy} 5-\mathrm{mas}_{3}$ provided the best uptake ratios, as relates to the faster clearance of this tracer from these tissues.

Fluorescence imaging of the tumor-containing prostate tissue with a clinical laparoscope for Cy5 imaging allowed for visual tumor identification (Fig. 4). In line with the brightness (Table 1), affinity (Figs. 2A-2E), and biodistribution (Supplemental Table 3),

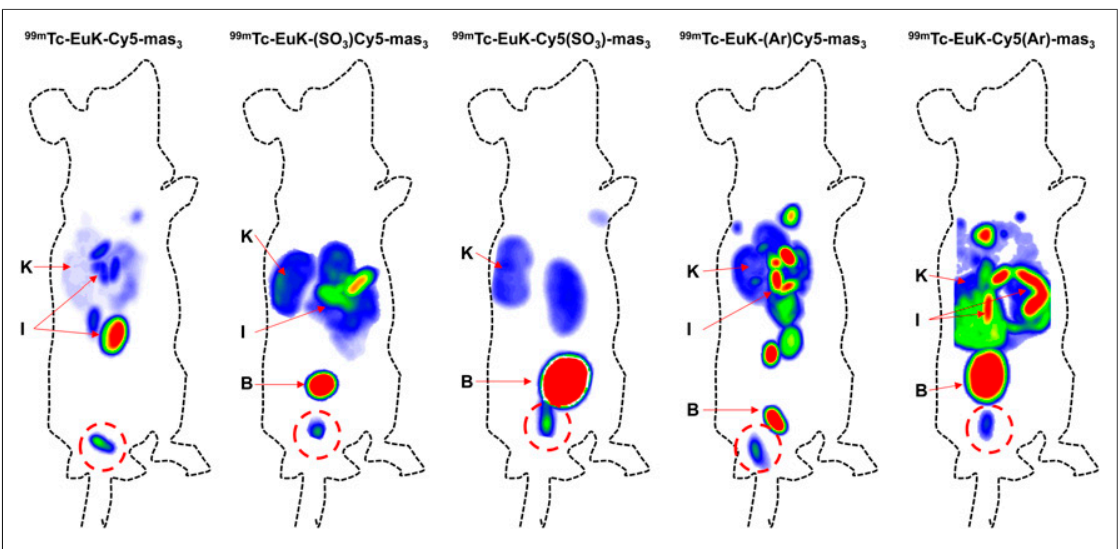

FIGURE 3. In vivo SPECT imaging evaluation using BALB/c nude mice with orthotopically transplanted PC346C cells, each injected with a different PSMA hybrid tracer analog. All images are scaled relative to tumor. $\mathrm{B}=$ bladder; I = intestines; $\mathrm{K}=$ kidneys. Tumor is encircled in red. the intensity of the fluorescence signal stresses that the introduction of an extra benzene moiety on the chelate-bearing indole slightly reduces the detectability of the lesion.

\section{DISCUSSION}

By exploring the molecular design of cyanine-based PSMA-targeted hybrid tracers, we found that changes in physical and photophysical properties, plasma protein interactions, and PSMA affinity could be related to in vivo tracer performance. Interestingly, the current findings indicate that a dye can also become an integral part of the pharmacophore. Of the evaluated panel of compounds, ${ }^{99 \mathrm{~m} T c-E u K-}\left(\mathrm{SO}_{3}\right) \mathrm{Cy} 5-\mathrm{mas}_{3}$ was the most favorable hybrid PSMA-targeted tracer.

In contrast to the introduction of a benzene moiety, the introduction of a sulfonate moiety induced a positive effect on the photophysical properties; the brightness increased by at least $27 \%$ compared with the nonsubstituted analog EuK-Cy5-mas 3 (Table 1). These findings are in line with previous reports on the photophysical properties of Cy5 and Cy7 analogs $(41,49)$. It should be noted that $\mathrm{Cy} 5$ analogs in general yield an approximately 2-fold higher brightness than comparative $\mathrm{Cy} 7$ analogs and are thus favored for use in optical in vivo imaging $(42,48)$.

Our findings are in concurrence with literature on radiotracer designs indicating that the location of chemical substituents in the backbone is of the utmost importance for interactions with PSMA's amphipathic funnel (Fig. 2) (12,13,33). Interestingly, the introduction of benzene moieties did not increase the affinity, whereas the introduction of an anionic sulfonate moiety on the $\mathrm{C}$ terminus of the cyanine backbone increased the PSMA affinity compared with EuK-Cy5-mas 3 . Furthermore, the affinity of EuK- $\left(\mathrm{SO}_{3}\right) \mathrm{Cy} 5$ $\operatorname{mas}_{3}$ was more than 9-fold higher than that of EuK-Cy5 $\left(\mathrm{SO}_{3}\right)$-mas ${ }_{3}$, suggesting interactions with the amphipathic entrance funnel; the cyanine backbone allows hydrophobic interactions to be complemented by hydrogen bonding enabled by careful placement of the sulfonate moiety (Fig. 2; Supplemental Fig. 12). The Cy5 dyes alone have a (calculated) lipophilicity ranging from a clogP of 1.4 to a clogP of 6.3 , which overlaps with the lipophilicity reported for favorable backbones that contain a naphthalic moiety (distribution coefficient, 2.9) (33). In previous studies, ${ }^{\text {nat }} \mathrm{Re}-\mathrm{labeled}$ analogs were chosen to determine a compound's affinity, because coordination of a metal ion to the chelate was shown to increase the PSMA affinity (20). A limitation of the current study is that these experiments with ${ }^{\text {nat }} \mathrm{Re}$ were not performed, meaning 


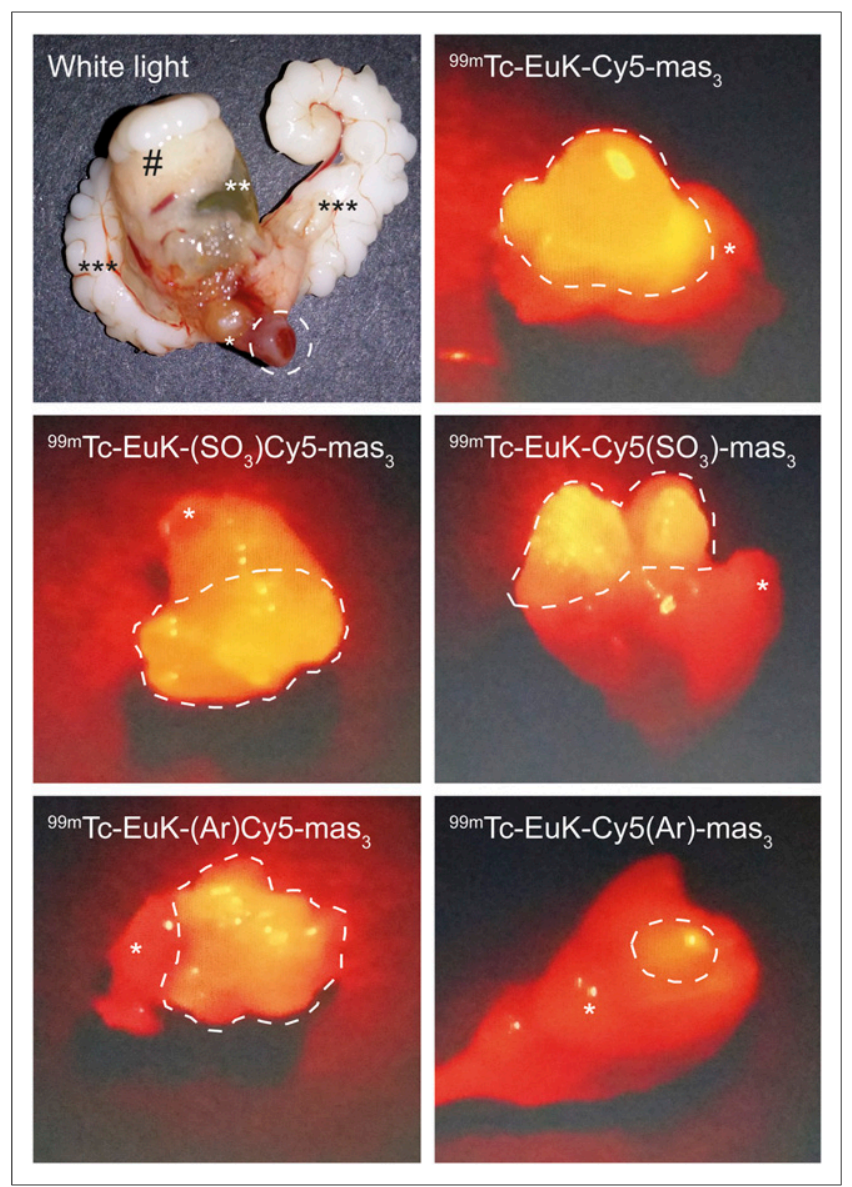

FIGURE 4. White-light and ex vivo fluorescence imaging of tumorcontaining prostate tissue $\left({ }^{\star}\right)$, bladder $\left(^{* \star}\right)$, seminal vesicles $\left({ }^{\star \star *}\right)$, and abdominal adipose tissue (\#) after injection of corresponding hybrid tracer.

the affinity of the compounds may in fact be higher than reported here. However, the trend between compound affinities is expected to be similar. The current setting also allowed us to make comparisons with literature values. Future studies of $k_{\text {on }}$ (association rate constant) and $k_{\text {off }}$ (dissociation rate constant) might provide a deeper understanding of the receptor affinity in relation to the tracer composition. Also, when $1 \mathrm{nmol}$ is applied to a mouse, only a relatively small portion of the tracer will be radiolabeled with ${ }^{99 \mathrm{~m}} \mathrm{Tc}$, meaning fluorescence guidance is not essentially dependent on the radiolabeled fraction of the tracer. The use of higher doses than, for example, 60 pmol-0.2 nmol (50), is substantiated by the fact that studies with fluorescent-only PSMA-targeted tracers containing cyanine dyes regularly use $1-10 \mathrm{nmol}(22,51)$.

Cyanine dyes, such as indocyanine green (a cyanine dye with 2 sulfonates and 2 benzene moieties), are well known for their albumin-binding properties (52). In line with this characteristic, the PPB increased when a sulfonate or extra benzene moiety was added onto the cyanine backbone (Table 1), molecular features that have been assigned to Sudlow site I or II (53). The stability measurements in serum have shown high stability for both imaging labels, thus complementing the literature showing that the EuK moiety is stable (9). The trends observed in lipophilicity and PPB were, however, only in part related to the biodistribution profile (Table 1; Supplemental Table 3). This observation stresses the important role that other molecular characteristics, such as steric hindrance, polar surface area, or pKa, play on tracer performance. The most lipophilic hybrid tracers (with an extra benzene moiety on either indole) provided an increased hepatobiliary clearance-which could potentially be a favorable feature-including increased intestinal uptake compared with the sulfonate-containing hybrid tracers, as is in line with the literature (47). Furthermore, the most lipophilic tracers presented an increased retention in muscle, blood, prostate, and fat at $2 \mathrm{~h}$ after injection, thus reducing tumor-to-muscle and tumor-to-blood ratios (Supplemental Table 3). The biodistribution data at the 2-h time point used during this study unfortunately complicates a direct comparison with studies that used a 1-h time point (such as for PSMA I\&F (31) or ${ }^{18} \mathrm{~F}-4(30)$ ), as biologic half-life can influence renal clearance. Given the delay between imaging and surgery, we did, however, find it necessary to take the 2-h time point to assess the tracer potential in an image-guided surgery setting.

Next to the molecular design and the ability to relate the tracer performance to a specific cyanine backbone structure, one of the clear differences between our study and other studies regarding PSMA-targeted small-molecule hybrid tracers is reduced splenic and renal uptake $(29-31,54)$. Considering that the renal uptake of DOTA (or DOTA analog)-containing PSMAtargeted hybrid tracers at early time points is around $100 \% \mathrm{ID} / \mathrm{g}$ or even higher $(29,54)$, whereas our study and the study of Kommidi et al. (30) yielded a renal uptake below $19 \% \mathrm{ID} / \mathrm{g}$, it seems that the introduction of radiolabels without relying on charged chelates in the design of hybrid PSMA-targeted tracers minimizes renal uptake. As literature underlines that the chelate composition can also have a profound effect on the biodistribution pattern (29), the insights now obtained could support further design of PSMA-targeted hybrid tracers.

In agreement with previous reports $(42,45,47)$, the recent biodistribution data underline once more that the composition of the cyanine backbone is of sheer importance in designing hybrid tracers and that asymmetric dye substitution holds promise during tracer refinement.

\section{CONCLUSION}

We have demonstrated that cyanine dyes can be used as a chemical backbone to connect an EuK targeting vector with a chelate for radiolabeling. By doing so, we discovered that the dye composition influences the tracer affinity by interacting with the amphipathic funnel of the PSMA protein. In particular, the introduction of a sulfonate moiety on the EuK-bearing indole allowed us to tune the affinity and in vivo performance. Overall, these efforts yielded a new PSMA-targeted hybrid tracer that could help accommodate the need for optical augmentation during PSMA-targeted radioguided surgery.

\section{DISCLOSURE}

Molecular graphics and analyses were performed with UCSF Chimera, which was developed by the Resource for Biocomputing, Visualization, and Informatics at the University of California, San Francisco, with support from NIH P41-GM103311. This research is funded by an NWO-TTW-VICI grant (TTW 16141). Fijs van Leeuwen is a consultant for Hamamatsu Photonics and is the chief innovation officer at ORSI Academy. Hans-Jürgen Wester is founder and shareholder of Scintomics GmbH. No 
other potential conflict of interest relevant to this article was reported.

\section{KEY POINTS}

QUESTION: Is it possible to make the fluorescent dye an integral part of the pharmacophore?

PERTINENT FINDINGS: This preclinical study portrayed the use of cyanine dyes as an integral part of the pharmacophore in the design of PSMA-targeted hybrid tracers.

IMPLICATIONS FOR PATIENT CARE: First, new hybrid tracer designs are required to advance the field of image-guided surgery. Second, reduction of renal retention of hybrid tracers might reduce hindrance of navigation in the surgical field caused by spillage of fluorescent urine. The promising findings suggest that clinical studies using PSMA I\&S may in the future benefit from a hybrid PSMA-tracer variant.

\section{REFERENCES}

1. Bray F, Ferlay J, Soerjomataram I, Siegel RL, Torre LA, Jemal A. Global cancer statistics 2018: GLOBOCAN estimates of incidence and mortality worldwide for 36 cancers in 185 countries. CA Cancer J Clin. 2018;68:394-424.

2. van Leeuwen FWB, Winter A, van Der Poel HG, et al. Technologies for imageguided surgery for managing lymphatic metastases in prostate cancer. Nat Rev Urol. 2019;16:159-171.

3. van Leeuwen FWB, van Oosterom MN, Meershoek P, et al. Minimal-invasive robot-assisted image-guided resection of prostate-specific membrane antigenpositive lymph nodes in recurrent prostate cancer. Clin Nucl Med. 2019;44:580581.

4. Freedland SJ, Humphreys EB, Mangold LA, et al. Risk of prostate cancer-specific mortality following biochemical recurrence after radical prostatectomy. JAMA. 2005;294:433-439.

5. Bostwick DG, Pacelli A, Blute M, Roche P, Murphy GP. Prostate specific membrane antigen expression in prostatic intraepithelial neoplasia and adenocarcinoma: a study of 184 cases. Cancer. 1998;82:2256-2261.

6. Lindenberg ML, Turkbey B, Mena E, Choyke PL. Imaging locally advanced, recurrent, and metastatic prostate cancer: a review. JAMA Oncol. 2017;3:14151422 .

7. Maurer T, Eiber M, Schwaiger M, Gschwend JE. Current use of PSMA-PET in prostate cancer management. Nat Rev Urol. 2016;13:226-235.

8. Weineisen M, Schottelius M, Šimeček J, Eiber M, Schwaiger M, Wester H. Development and first in human evaluation of PSMA I\&T: a ligand for diagnostic imaging and endoradiotherapy of prostate cancer [abstract]. $\mathrm{J} \mathrm{Nucl} \mathrm{Med}$. 2014;55(suppl):1083.

9. Weineisen M, Simecek J, Schottelius M, Schwaiger M, Wester HJ. Synthesis and preclinical evaluation of DOTAGA-conjugated PSMA ligands for functional imaging and endoradiotherapy of prostate cancer. EJNMMI Res. 2014;4:63.

10. Ferraris DV, Shukla K, Tsukamoto T. Structure-activity relationships of glutamate carboxypeptidase II (GCPII) inhibitors. Curr Med Chem. 2012;19:12821294.

11. Mesters JR, Barinka C, Li W, et al. Structure of glutamate carboxypeptidase II, a drug target in neuronal damage and prostate cancer. EMBO J. 2006;25:13751384 .

12. Maung J, Mallari JP, Girtsman TA, et al. Probing for a hydrophobic a binding register in prostate-specific membrane antigen with phenylalkylphosphonamidates. Bioorg Med Chem. 2004;12:4969-4979.

13. Baranski AC, Schäfer M, Bauder-Wüst U, et al. Improving the imaging contrast of ${ }^{68} \mathrm{Ga}$-PSMA-11 by targeted linker design: charged spacer moieties enhance the pharmacokinetic properties. Bioconjug Chem. 2017;28:2485-2492.

14. Kuo HT, Pan J, Zhang Z, et al. Effects of linker modification on tumor-to-kidney contrast of ${ }^{68} \mathrm{Ga}$-labeled PSMA-targeted imaging probes. Mol Pharm. 2018;15: 3502-3511.

15. Liu T, Nedrow-Byers JR, Hopkins MR, Berkman CE. Spacer length effects on in vitro imaging and surface accessibility of fluorescent inhibitors of prostate specific membrane antigen. Bioorg Med Chem Lett. 2011;21:7013-7016.

16. Heck MM, Tauber R, Schwaiger S, et al. Treatment outcome, toxicity, and predictive factors for radioligand therapy with ${ }^{177} \mathrm{Lu}$-PSMA-I\&T in metastatic castration-resistant prostate cancer. Eur Urol. 2019;75:920-926.
17. Kratochwil C, Giesel FL, Stefanova M, et al. PSMA-targeted radionuclide therapy of metastatic castration-resistant prostate cancer with ${ }^{177} \mathrm{Lu}$-labeled PSMA617. J Nucl Med. 2016;57:1170-1176.

18. Horn T, Kronke M, Rauscher I, et al. Single lesion on prostate-specific membrane antigen-ligand positron emission tomography and low prostate-specific antigen are prognostic factors for a favorable biochemical response to prostate-specific membrane antigen-targeted radioguided surgery in recurrent prostate cancer. Eur Urol. 2019;76:517-523.

19. van Leeuwen FWB, van Oosterom MN, Meershoek P, et al. Minimal-invasive robot-assisted image-guided resection of prostate-specific membrane antigenpositive lymph nodes in recurrent prostate cancer. Clin Nucl Med. 2019;44: $580-581$.

20. Robu S, Schottelius M, Eiber M, et al. Preclinical evaluation and first patient application of Tc-99m-PSMA-I\&S for SPECT imaging and radioguided surgery in prostate cancer. J Nucl Med. 2017;58:235-242.

21. Rosenthal EL, Warram JM, de Boer E, et al. Successful translation of fluorescence navigation during oncologic surgery: a consensus report. J Nucl Med. 2016; 57:144-150.

22. Chen Y, Dhara S, Banerjee SR, et al. A low molecular weight PSMA-based fluorescent imaging agent for cancer. Biochem Biophys Res Commun. 2009;390: $624-629$.

23. Kularatne SA, Thomas M, Myers $\mathrm{CH}$, et al. Evaluation of novel prostate-specific membrane antigen-targeted near-infrared imaging agent for fluorescence-guided surgery of prostate cancer. Clin Cancer Res. 2019;25:177-187.

24. Liu T, Wu LY, Hopkins MR, Choi JK, Berkman CE. A targeted low molecular weight near-infrared fluorescent probe for prostate cancer. Bioorg Med Chem Lett. 2010;20:7124-7126.

25. Chen Y, Pullambhatla M, Banerjee SR, et al. Synthesis and biological evaluation of low molecular weight fluorescent imaging agents for the prostate-specific membrane antigen. Bioconjug Chem. 2012;23:2377-2385.

26. Matsuoka D, Watanabe H, Shimizu Y, et al. Structure-activity relationships of succinimidyl-Cys-C(O)-Glu derivatives with different near-infrared fluorophores as optical imaging probes for prostate-specific membrane antigen. Bioorg Med Chem. 2018;26:2291-2301.

27. KleinJan GH, Bunschoten A, van den Berg NS, et al. Fluorescence guided surgery and tracer-dose, fact or fiction? Eur J Nucl Med Mol Imaging. 2016;43: 1857-1867.

28. van Leeuwen FW, Valdés-Olmos RA, Buckle T, Vidal-Sicart S. Hybrid surgical guidance based on the integration of radionuclear and optical technologies. $\mathrm{Br}$ J Radiol. 2016;89:20150797.

29. Banerjee SR, Pullambhatla M, Byun Y, et al. Sequential SPECT and optical imaging of experimental models of prostate cancer with a dual modality inhibitor of the prostate-specific membrane antigen. Angew Chem Int Ed Engl. 2011;50: 9167-9170.

30. Kommidi H, Guo H, Nurili F, et al. ${ }^{18} \mathrm{~F}$-positron emitting/trimethine cyaninefluorescent contrast for image-guided prostate cancer management. J Med Chem. 2018;61:4256-4262.

31. Schottelius M, Wurzer A, Wissmiller K, et al. Synthesis and preclinical characterization of the PSMA-targeted hybrid tracer PSMA-I\&F for nuclear and fluorescence imaging of prostate cancer. J Nucl Med. 2019;60:71-78.

32. van Leeuwen FWB, van der Poel HG. Surgical guidance in prostate cancer: "from molecule to man" translations. Clin Cancer Res. 2016;22:1304-1306.

33. Benešová $M$, Bauder-Wüst $U$, Schäfer $M$, et al. Linker modification strategies to control the prostate-specific membrane antigen (PSMA)-targeting and pharmacokinetic properties of DOTA-conjugated PSMA inhibitors. J Med Chem. 2016; 59:1761-1775.

34. Pedersen DS, Rosenbohm C. Dry column vacuum chromatography. SynthesisStuttgart. 2001;2001:2431-2434.

35. Lopalco M, Koini EN, Cho JK, Bradley M. Catch and release microwave mediated synthesis of cyanine dyes. Org Biomol Chem. 2009;7:856-859.

36. Khan TH, Eno-Amooquaye EA, Searle F, Browne PJ, Osborn HM, Burke PJ. Novel inhibitors of carboxypeptidase G2 (CPG2): potential use in antibody-directed enzyme prodrug therapy. J Med Chem. 1999;42:951-956.

37. Makowski M, Rzeszotarska B, Smelka L, Kubica Z. Synthesis of peptides with $\alpha, \beta$-dehydroamino acids, III. Debenzyloxycarbonylation and detrifluoroacetylation of dehydroalanine and dehydrophenylalanine peptides. Liebigs Annalen der Chemie. 1985;1985:1457-1464.

38. Mujumdar RB, Ernst LA, Mujumdar SR, Lewis CJ, Waggoner AS. Cyanine dye labeling reagents: sulfoindocyanine succinimidyl esters. Bioconjug Chem. 1993;4: $105-111$.

39. Sauer M, Hofkens J, Enderlein J. Basic principles of fluorescence spectroscopy. In: Sauer M, Hofkens J, Enderlein J, et al., eds. Handbook of Fluorescence Spectroscopy and Imaging. Weinheim, Germany: Wiley; 2011:1-30. 
40. Spa SJ, Hensbergen AW, van der Wal S, Kuil J, van Leeuwen FWB. The influence of systematic structure alterations on the photophysical properties and conjugation characteristics of asymmetric cyanine 5 dyes. Dyes Pigments. 2018;152: 19-28.

41. van der Wal S, Kuil J, Valentijn ARPM, van Leeuwen FWB. Synthesis and systematic evaluation of symmetric sulfonated centrally $\mathrm{C} \mathrm{C}$ bonded cyanine near-infrared dyes for protein labelling. Dyes Pigments. 2016;132:7-19.

42. Buckle T, van Willigen DM, Spa SJ, et al. Tracers for fluorescence-guided surgery: how elongation of the polymethine chain in cyanine dyes alters the pharmacokinetics of a dual-modality c[RGDyK] tracer. J Nucl Med. 2018;59: 986-992.

43. Trott O, Olson AJ. AutoDock Vina: improving the speed and accuracy of docking with a new scoring function, efficient optimization, and multithreading. $J$ Comput Chem. 2010;31:455-461.

44. Pettersen EF, Goddard TD, Huang CC, et al. UCSF Chimera: a visualization system for exploratory research and analysis. J Comput Chem. 2004;25:16051612 .

45. Hensbergen AW, van Willigen DM, Welling MM, et al. Click chemistry in the design and production of hybrid tracers. ACS Omega. 2019;4:12438-12448.

46. Marques RB, van Weerden WM, Erkens-Schulze S, et al. The human PC346 xenograft and cell line panel: a model system for prostate cancer progression. Eur Urol. 2006;49:245-257.
47. Bunschoten A, van Willigen DM, Buckle T, et al. Tailoring fluorescent dyes to optimize a hybrid RGD-tracer. Bioconjug Chem. 2016;27:1253-1258.

48. van Willigen DM, van den Berg NS, Buckle T, et al. Multispectral fluorescence guided surgery; a feasibility study in a phantom using a clinical-grade laparoscopic camera system. Am J Nucl Med Mol Imaging. 2017;7:138-147.

49. Wirtz M, Schmidt A, Schottelius M, et al. Synthesis and in vitro and in vivo evaluation of urea-based PSMA inhibitors with increased lipophilicity. EJNMMI Res. 2018;8:84.

50. Kung MP, Kung HF. Mass effect of injected dose in small rodent imaging by SPECT and PET. Nucl Med Biol. 2005;32:673-678.

51. Kwon YD, Chung HJ, Lee SJ, Lee SH, Jeong BH, Kim HK. Synthesis of novel multivalent fluorescent inhibitors with high affinity to prostate cancer and their biological evaluation. Bioorg Med Chem Lett. 2018;28:572-576.

52. Bunschoten A, Buckle T, Kuil J, et al. Targeted non-covalent self-assembled nanoparticles based on human serum albumin. Biomaterials. 2012;33:867875 .

53. Buckle T, Chin PT, van Leeuwen FW. (Non-targeted) radioactive/fluorescent nanoparticles and their potential in combined pre- and intraoperative imaging during sentinel lymph node resection. Nanotechnology. 2010;21:482001.

54. Baranski AC, Schäfer M, Bauder-Wüst U, et al. PSMA-11-derived dual-labeled PSMA inhibitors for preoperative PET imaging and precise fluorescence-guided surgery of prostate cancer. $J$ Nucl Med. 2018;59:639-645. 